\title{
Physiological responses of Pseudomonas aeruginosa PAO1 to oxidative stress in controlled microaerobic and aerobic cultures
}

\author{
Wael Sabra, Eun-Jin Kim and An-Ping Zeng
}

GBF - Gesellschaft für Biotechnologische

Forschung $\mathrm{mbH}$, Microbial Systems, Mascheroder Weg 1, 38124 Braunschweig, Germany
Author for correspondence: An-Ping Zeng. Tel: +495316181 188. Fax: +49 5316181751. e-mail: AZE@GBF.de

\begin{abstract}
Pseudomonas aeruginosa PAO1 was found to exhibit several remarkable physiological responses to oxidative stress upon its growth in a computercontrolled suspension culture. First, it strongly reduced the transfer rate of oxygen from the gas into the liquid phase, causing oxygen-limited or microaerophilic conditions in the culture after a short period of cultivation, even at high aeration rates with pure oxygen. Second, PAO1 that was previously classified as 'non-mucoid' formed a clear polysaccharide capsule on the cell surface (mucoid phenotype) under oxidative-stress conditions. Third, the strain showed a reduced growth rate and a longer lag phase under high oxygen tension. Finally, $P$. aeruginosa PAO1 released a high amount of proteins into the culture broth. The release of some virulence factors by PAO1, such as elastase, was significantly enhanced or only occurred under microaerobic conditions (i.e. dissolved oxygen tension value around $1 \%$ of air saturation). Hence, it is concluded that $P$. aeruginosa PAO1 prefers microaerobic conditions for growth and for the formation of some of its virulence factors. PAO1 can create such growth conditions by at least two mechanisms: (i) blockage of the transfer of oxygen and (ii) formation of a polysaccharide capsule on the cell surface. It is postulated that the blockage of oxygen transfer may play an important role in the defence of this pathogen against reactive oxygen intermediates.
\end{abstract}

Keywords: oxygen transfer, pathophysiology, polysaccharide capsule, microaerophilic growth, virulence factor

\section{INTRODUCTION}

Pseudomonas aeruginosa is the major pathogen in chronic pulmonary infections of patients with cystic fibrosis (CF) (Pier, 1998). CF is the most common human lethal recessively-transmitted disease and is caused by mutations in a gene encoding the $\mathrm{CF}$ transmembrane conductance regulator, which channels chloride ions to the apical membrane of involved epithelia (Goldberg \& Pier, 2000; Govan \& Deretic, 1996). The disease is characterized by bacterial colonization and chronic airway infection that progressively destroys the lungs of patients and often leads to respiratory failure. The strains of $P$. aeruginosa that initially infect the lungs of CF patients have a non-

Abbreviations: $\mathrm{CF}$, cystic fibrosis; $k_{\mathrm{L}} a$, oxygen transfer coefficient; $\mathrm{pO}_{2^{\prime}}$ dissolved oxygen tension. mucoid phenotype that is typical of isolates that occur in the environment. These strains are also highly motile and secrete high levels of protease exotoxins and siderophores (Deretic et al., 1993; Mathee et al., 1999; Zaborina et al., 2000). After the initial infection of CF patients by $P$. aeruginosa, the organism undergoes a number of important morphological changes that give rise to mucoidy (Kharazmi, 1991; Mathee et al., 1999). The production of alginate by the bacterium and the formation of microcolonies in the lungs of patients are the most characteristic features of persistent $P$. aeruginosa infection. This microcolony form of growth (biofilm) represents a survival strategy of the bacterium in chronic $P$. aeruginosa infections. Mucoid $P$. aeruginosa strains have an advantage over non-mucoid strains, in that they are partially protected against phagocytosis, antibodies and antibiotic treatment (Govan \& Harris, 1986; Greenberg, 2000; Ishida et al., 1998). Mucoid strains of $P$. aeruginosa from chronic 
lung infections in CF patients are generally non-motile and express lower levels of protease exotoxins and siderophores than non-mucoid strains (Govan \& Deretic, 1996).

Numerous cell-associated factors, including alginate, pili and lipopolysaccharide, are important in $P$. aeruginosa virulence. Furthermore, many $P$. aeruginosa virulence factors, including toxins (exotoxin A and exoenzyme S), proteases (elastase, LasA protease and alkaline protease) and haemolysins (phospholipase and rhamnolipid), are released from cells of the organism (Kamath et al., 1998; Rumbaugh et al., 1999). These factors contribute to the virulence of $P$. aeruginosa in animal models and have been the focus of in vitro studies with clinical isolates (Pearson et al., 1997).

The pathogenic roles of alginate, in particular in relation to the formation of the mucoid phenotype in $P$. aeruginosa strains, have received much attention (see review by Govan \& Deretic, 1996). It is now recognized that oxygen plays an important role in the formation of alginate and the appearance of the mucoid phenotype in P. aeruginosa (Krieg et al., 1986; Leitäo \& Sä-Correia, 1997; Sato et al., 1984; Xu et al., 1998). Oxygendependent upregulation of transcription of alginate genes was normally found in highly mucoid strains of $P$. aeruginosa (Leitäo \& Sä-Correia, 1997). In an experiment with a mixture of mucoid and non-mucoid $P$. aeruginosa strains (1:1), Krieg et al. (1986) found that aeration of cultures favourably selected for the growth of mucoid strains. Non-mucoid strains of $P$. aeruginosa were reported to be sensitive to oxygen. In previous studies with another alginate-producing bacterium $(A z-$ otobacter vinelandii) grown under controlled oxygen tension in a bioreactor system, we have shown that the formation of alginate exopolysaccharide on the cell surface of this organism is dependent on the oxygen tension (Sabra et al., 2001, 1999). Hence, alginate formation represents an important mechanism for protecting bacterial cells from oxidative stress, especially under conditions of diazotrophical growth.

In this work, we have quantitatively studied the physiological responses of $P$. aeruginosa PAO1 to oxidative stress in computer-controlled microaerobic and aerobic cultures. In addition to cell growth, attention was paid to polysaccharide formation and the release of proteins (virulence factors) by the bacterium under these conditions. For the first time, we report that cultures of $P$. aeruginosa PAO1 exhibit the unusual property of dramatically reducing the efficiency of oxygen transfer and thus the dissolved oxygen concentration in the culture broth. The reasons and consequences for these unusual physiological responses to oxidative stress are discussed.

\section{METHODS}

Micro-organism and cultivation conditions. $P$. aeruginosa PAO1 was grown under varying dissolved oxygen tension $\left(p \mathrm{O}_{2}\right)$ levels in a computer-controlled bioreactor. Glucose minimal medium (Mian et al., 1978) was used for growth of the organism with slight modifications [all components were added (l distilled water $\left.)^{-1}\right]$ : glucose, $30 \mathrm{~g} ;\left(\mathrm{NH}_{4}\right)_{2} \mathrm{SO}_{4}$, $2 \mathrm{~g} ; \mathrm{K}_{2} \mathrm{HPO}_{4}, \quad 0.22 \mathrm{~g} ; \mathrm{KH}_{2} \mathrm{PO}_{4}, 0.08 \mathrm{~g} ; \mathrm{NaCl}, 0.2 \mathrm{~g}$; $\mathrm{MgSO}_{4} .7 \mathrm{H}_{2} \mathrm{O}, 0.2 \mathrm{~g} ; \mathrm{CaCl}_{2} .2 \mathrm{H}_{2} \mathrm{O}, 0.05 \mathrm{~g} ; \mathrm{ZnSO}_{4} .7 \mathrm{H}_{2} \mathrm{O}$, $0.2 \mathrm{mg} ; \mathrm{CuSO}_{4} .5 \mathrm{H}_{2} \mathrm{O}, 0.2 \mathrm{mg} ; \mathrm{MnSO}_{4} . \mathrm{H}_{2} \mathrm{O}, 0.2 \mathrm{mg} ; \mathrm{CoCl}_{2}$, $0.2 \mathrm{mg} ; \mathrm{FeSO}_{4} .7 \mathrm{H}_{2} \mathrm{O}, 0.6 \mathrm{mg}$. Maintenance medium A (Mian et al., 1978) was used for seed cultivation.

A shake-flask-culture study was done in $100 \mathrm{ml}$ Erlenmeyer flasks containing $40 \mathrm{ml}$ medium A (Mian et al., 1978). In experiments for evoking mucoidy in PAO1 by oxidative stress, the cells were exposed to $30 \mathrm{mM} \mathrm{H}_{2} \mathrm{O}_{2}$ in the early-exponential phase of growth and shaken vigorously at $37^{\circ} \mathrm{C}$.

Bioreactor and control. Cultivation of PAO1 was carried out in a 2.51 stirred tank bioreactor (Applikon) with a working volume of $1.5 \mathrm{l}$. The agitation speed was kept constant at 500 r.p.m. by a proportional-integral-differential (PID) controller installed in the control unit of the bioreactor. Temperature was kept at $37^{\circ} \mathrm{C}$ by a single-loop PID controller. An autoclavable $\mathrm{pH}$ electrode (Mettler-Toledo) was used to measure the $\mathrm{pH}$, which was kept at $7 \cdot 0 \pm 0 \cdot 2$ by the automatic addition of $\mathrm{HCl}(2 \mathrm{M})$ or $\mathrm{NaOH}(2 \mathrm{M})$ to the bioreactor. The $p \mathrm{O}_{2}$ was measured by using an autoclavable $p \mathrm{O}_{2}$ electrode (Mettler-Toledo).

The bioreactor was aerated with a fine-porous gas distributor. Thermal mass flow meters/controllers were used for the supply of gases. The total aeration rate was kept at a constant value $\left(1.51 \mathrm{~min}^{-1}\right)$ by the real-time operation computer control system UBICON (Universal Bioprocess Control System, ESD, Hanover, Germany). $p \mathrm{O}_{2}$ was controlled over the range $1-50 \pm 1 \cdot 5 \%$ of air saturation by mixing nitrogen and pure oxygen in the inlet gas using a PID controller defined through the UBICON facilities.

Analytical methods. Biomass dry weight of PAO1 was determined gravimetrically as described previously (Sabra $e t$ al., 2000). The alginate concentration in the culture supernatant was determined by precipitation of the compound with 3 vols of ice cold 2-propanal. The alginate pellet was suspended in water, reprecipitated with 2-propanal and resuspended in $2.5 \mathrm{ml}$ water. The amount of alginate recovered from the culture supernatant was determined using a borate/ carbazole method (Knutson \& Jeanes, 1968), with algal alginate used for calibration of the standard curve.

The total extracellular protein content of the cell-free supernatant was determined by using the Lowry method. Elastase activity present in the cell-free supernatant was determined in a spectrophotometric assay using Elastin/Congo red (Sigma) as a substrate, as described by Kessler et al. (1993). The amount of rhamnolipid present in the cell-free supernatant was determined by a duplicate orcinol assay compared to a rhamnose standard (Kock et al., 1991). Rhamnolipid was determined by using the relationship $0.1 \mathrm{mg}$ of rhamnose corresponds to $2.5 \mathrm{mg}$ of rhamnolipid.

Electron microscopy. Transmission electron microscopy was used to detect the alginate capsule on the cell surface of $P$. aeruginosa cells that had been taken directly from the controlled bioreactor or uncontrolled flask cultures. Embedding and ultra-thin sectioning of $P$. aeruginosa were done as described previously for A. vinelandii (Sabra et al., 2000).

Oxygen transfer coefficient $\left(k_{\mathrm{L}} a\right)$ determination. For determination of the volumetric $k_{\mathrm{L}} a$, the static method of gassing-out was used (Stanbury \& Whitaker, 1987). The equation applied for the determination of the $k_{\mathrm{L}} a$ is as follows :

$\ln \left(C^{*}-C_{\mathrm{L}}\right)=-k_{\mathrm{L}} a t$ 
where $C^{*}$ is the oxygen saturation concentration of the liquid with the aeration gas, $C_{\mathrm{L}}$ is the concentration of oxygen in the liquid and $t$ is the time in seconds.

Cell-free culture supernatants contained within $50 \mathrm{ml}$ flasks were first sparged with nitrogen to get a $p \mathrm{O}_{2}$ value of zero; air was then supplied to the supernatants at a constant aeration rate and a constant agitation. An oxygen tension electrode connected to the UBICON system monitored the change in the dissolved oxygen concentration in the liquid. The increase in the dissolved oxygen concentration can be described by the above equation related with $k_{\mathrm{L}} a$. Temperature as well as agitation speed was fixed for all of the samples. The mean results of at least two measurements are presented.

\section{RESULTS}

\section{Control of $\mathrm{pO}_{2}$ and unusual properties of the culture broth of $P$. aeruginosa PAO1 with respect to oxygen transfer}

To quantitatively investigate the effects of $p \mathrm{O}_{2}$ on the growth and metabolism of $P$. aeruginosa PAO1, we cultivated this strain in a defined minimal medium at different $p \mathrm{O}_{2}$ values, i.e. $1,5,10$ or $50 \%$ of air saturation. The $p \mathrm{O}_{2}$ profiles and biomass formation of PAO1 during these cultivation processes are shown in Figs 1 and 2. Surprisingly, it was only possible to control the $\mathrm{pO}_{2}$ at the preset value during the first few hours of cultivation. Despite vigorous aeration of the bioreactor with pure oxygen in the late phase of growth (Fig. 1a), strong oxygen limitation occurred $6-8 \mathrm{~h}$ after inoculation of all of the cultures (Fig. 1b). This oxygen limitation was not caused by the presence of a high biomass concentration,

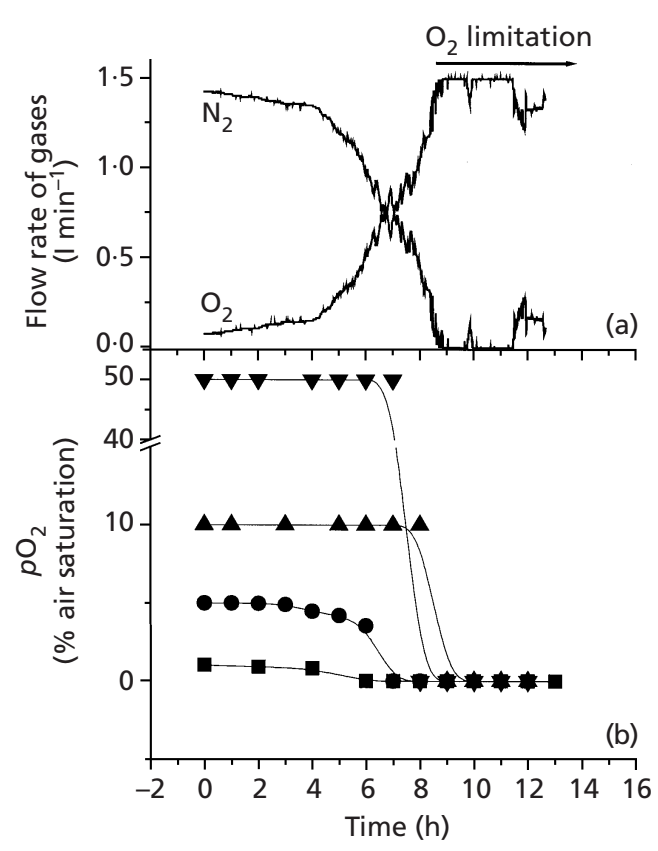

Fig. 1. (a) Flow rate $\left(1 \mathrm{~min}^{-1}\right)$ of aeration gases measured online for the control of the $\mathrm{pO}_{2}$ at $10 \%$ of air saturation, and (b) $\mathrm{pO}_{2}$ profile in experiments with $\mathrm{pO}_{2}$ levels set at $1 \%(\boldsymbol{\square}), 5 \%$ $(\boldsymbol{\bullet}), 10 \%(\boldsymbol{\Delta})$ and $50 \%(\boldsymbol{\nabla})$ of air saturation.

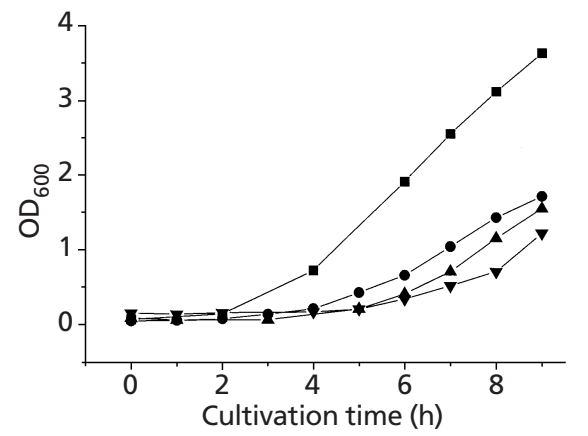

Fig. 2. Cell growth of $P A O 1$ as a function of the different $p \mathrm{O}_{2}$ levels tested. $\mathrm{pO}_{2}$ Values are expressed as a percentage of air saturation: $\mathbf{\square}, 1 \% ; 0,5 \% ; \boldsymbol{\Delta}, 10 \% ; \boldsymbol{\nabla}, 50 \%$.

as the amount of biomass produced was relatively low (maximum 0.4-1.6 $\mathrm{g}_{\text {biomass }} \mathrm{l}^{-1}$ ) (Fig. 2). It was also not caused by an unusually high level of oxygen consumption by the cells, since the dissolved oxygen concentration in the culture was almost zero under these conditions. Under fully aerobic conditions, $P$. aeruginosa normally has a specific oxygen uptake rate comparable to that of many other bacteria (Geckil et al., 2001). The same reactor set-up as used here can sustain an active aerobic growth of more than $10 \mathrm{~g}$ biomass $1^{-1}$ for organisms such as Saccharomyces cerevisiae without using pure oxygen aeration (unpublished data).

Therefore, the oxygen limitation observed in the $P$. aeruginosa $\mathrm{PAO} 1$ cultures must be due to a restriction in the transfer of oxygen from the gas phase to the cells. Since the dissolved oxygen concentration in the liquid phase was almost zero, the strong oxygen limitation could only be caused by a restriction on oxygen transfer from the gas phase into the liquid phase. To characterize oxygen transport through the gas/liquid interface, we measured the volumetric $k_{\mathrm{L}} a$, a key parameter for the rate of oxygen transfer (Bailey \& Ollis, 1985). Fig. 3 shows the results of measurements taken from the culture supernatant from one of the cultivations $\left(p \mathrm{O}_{2}\right.$ set value $=5 \%$ of air saturation); the $k_{\mathrm{L}} a$ value of the medium before inoculation with PAO1 is also shown in Fig. 3. Inoculation of the medium with PAO1 $5 \%$ of the working volume) led to an immediate decrease in the $k_{\mathrm{L}} a$ value $\left(50 \%\right.$ decrease). The $k_{\mathrm{L}} a$ value recovered to a certain extent during the first few hours of bacterial growth but decreased sharply shortly before the onset of oxygen limitation. Similar results were also obtained with culture supernatants obtained from other cultivation runs.

The very low $k_{\mathrm{L}} a$ value observed in the late phase of growth was not expected, but it could explain the immediate decrease in the $k_{\mathrm{L}} a$ value seen after inoculation of the medium with PAO1 and at the onset of oxygen limitation. The possible effects of biomass itself on the oxygen transfer rate were also examined. The $k_{\mathrm{L}} a$ value of a culture broth with dead cells was found to be similar to that of the supernatant without biomass, 


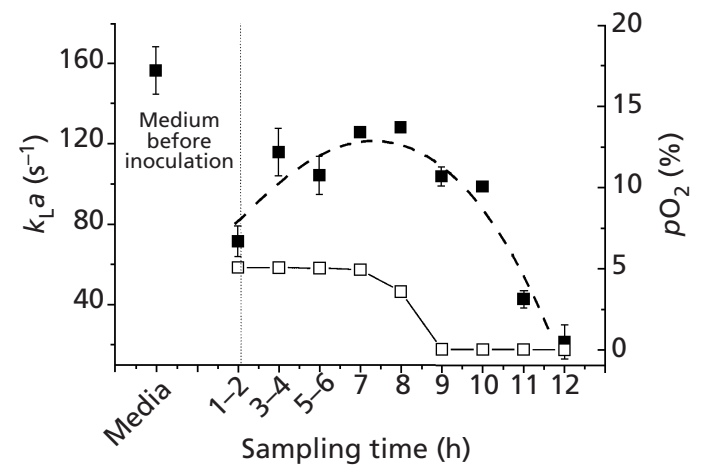

Fig. 3. Oxygen transfer coefficient $k_{\mathrm{L}} a(\boldsymbol{\sigma})$ in medium and culture supernatant and $\mathrm{pO}_{2}(\square)$ profile during cultivation of PAO1 with $\mathrm{pO}_{2}$ preset at $5 \%$ of air saturation.

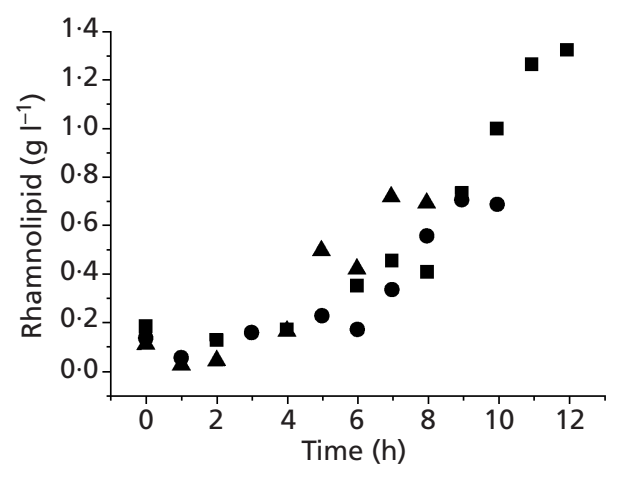

Fig. 4. Formation of rhamnolipid by batch cultures of $P$. aeruginosa PAO1 grown at different $\mathrm{pO}_{2}$ levels. $\square, 1 \%$ of air saturation; $\boldsymbol{C}, 10 \%$ of air saturation; $\boldsymbol{\Delta}, 50 \%$ of air saturation.

excluding a possible direct effect of biomass on the mass transfer. A similarly strong reduction in the $k_{\mathrm{L}} a$ value could be evoked in an uncontrolled (i.e. no $\mathrm{pO}_{2}$ control) flask culture of PAO1 by treatment with $\mathrm{H}_{2} \mathrm{O}_{2}$ (data not shown).

The reason for the strong reduction in the oxygen transfer rate in cultures of $P$. aeruginosa PAO1 is not clear. It is possible that this phenomenon could be due to the production of biosurfactants by $P$. aeruginosa. $P$. aeruginosa is known to produce several kinds of amphiphilic compounds, such as rhamnolipid, under oxidative stress. The production of these compounds can result in heavy foam formation and in significant alterations in the surface and interfacial tensions of water (Chayabutra et al., 2001; Guerra-Santos et al., 1986; Haba et al., 2000; Kock et al., 1991; Maier \& Soberon-Chavez, 2000; Rocha et al., 1992). The hydrophobic part of these amphiphilic compounds can bind onto the gas bubble, thereby forming an extra boundary layer and reducing the availability of oxygen for transfer to the bacterial cells. To assess this issue, we measured the level of rhamnolipid production in three $P$. aeruginosa cultures (Fig. 4). The amounts of rhamnolipid produced by the cultures increased successively in all three cultures and reached more than $1 \mathrm{~g}$ rhamnolipid $1^{-1}$. The specific formation rate of rhamnolipid was greater for cells grown at higher $p \mathrm{O}_{2}$ values. However, the absolute amount of rhamnolipid produced by the cultures was not significantly affected by the concentration of dissolved oxygen (Fig. 4). This is also in agreement with the results of $k_{\mathrm{L}} a$ measurements taken for supernatants from all of the cultures tested (Fig. 3). Whatever the mechanism involved in the reduction of oxygen transfer, it can be stated that this reduction of transfer in the culture broth is a general property of $P$. aeruginosa PAO1 cultures when grown under the experimental conditions applied in this work.

\section{Cell growth under different $\mathrm{pO}_{2}$ levels}

As shown in Fig. 2, P. aeruginosa PAO1 prefers to grow under microaerobic conditions. Biomass formation by the organism was considerably higher at $p \mathrm{O}_{2}=1 \%$ of air saturation than at $p \mathrm{O}_{2}=50 \%$ of air saturation. When the period during which the $p \mathrm{O}_{2}$ value was controlled at the set value is taken into consideration, the final biomass concentration of PAO1 varied inversely in relation to the increase in the $p \mathrm{O}_{2}$ value (data from Fig. 2, but not shown directly). At $p \mathrm{O}_{2}=1 \%$ of air saturation there was almost no lag phase in PAO1 growth, whereas the duration of the lag phase increased to about $4 \mathrm{~h}$ at $p \mathrm{O}_{2}=50 \%$ of air saturation. It was also found that the lower the $p \mathrm{O}_{2}$ value, the higher the specific growth rate, underlining the preference of $P$. aeruginosa $\mathrm{PAO} 1$ for microaerobic conditions.

\section{Occurrence of polysaccharide capsules on PAO1 cells as a response to oxidative stress}

$P$. aeruginosa PAO1 has been reported to be a nonmucoid clinical strain (Mathee et al., 1999). We investigated the possible appearance of a polysaccharide layer on the surface of PAO1 cells grown under different $p \mathrm{O}_{2}$ levels, by examining ultra-thin sections of negativelystained PAO1 cells using transmission electron microscopy. No obvious mucoid polysaccharide layer was observed on cells taken from a shake-flask culture (after $20 \mathrm{~h}$ incubation) (Fig. 5a). It is worth mentioning that an on-line measurement of $\mathrm{OO}_{2}$ values during shake-flask cultivation of $P$. aeruginosa $\mathrm{PAO} 1$ revealed oxygenlimited growth (i.e. $p \mathrm{O}_{2}=0$ ) of the organism 3-4 h after inoculation of the medium (data not shown). Since almost all of the work reported in the literature for $P$. aeruginosa PAO1 has been done using shake-flask cultures, the observed non-mucoidy of this strain may reflect a phenotype of this organism only seen when it is grown under oxygen-limited conditions. In fact, cells of PAO1 grown under controlled oxygen tension displayed a markedly different morphology to those grown under no oxygen control. The cells grown under controlled oxygen tension had a clear thick polysaccharide layer on their surfaces (Fig. 5b). A low amount of alginate $\left(0 \cdot 1-0 \cdot 3 \mathrm{~g}\right.$ alginate $\left.\mathrm{l}^{-1}\right)$ was also detected in the culture supernatant of strain PAO1 when it was grown under 

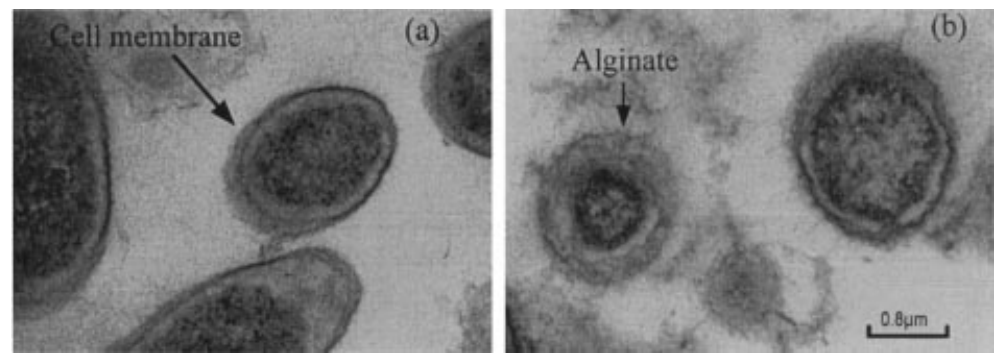

Fig. 5. Transmission electron micrographs of ultra-thin sections of negatively-stained vegetative cells of PAO1 grown under different conditions. (a) Cells of PAO1 from shake-flask culture $\left(\mathrm{pO}_{2}=0\right)$; (b) cells of PAO1 grown under controlled oxygen tension $\left(\mathrm{pO}_{2}=10 \%\right.$ of air saturation).
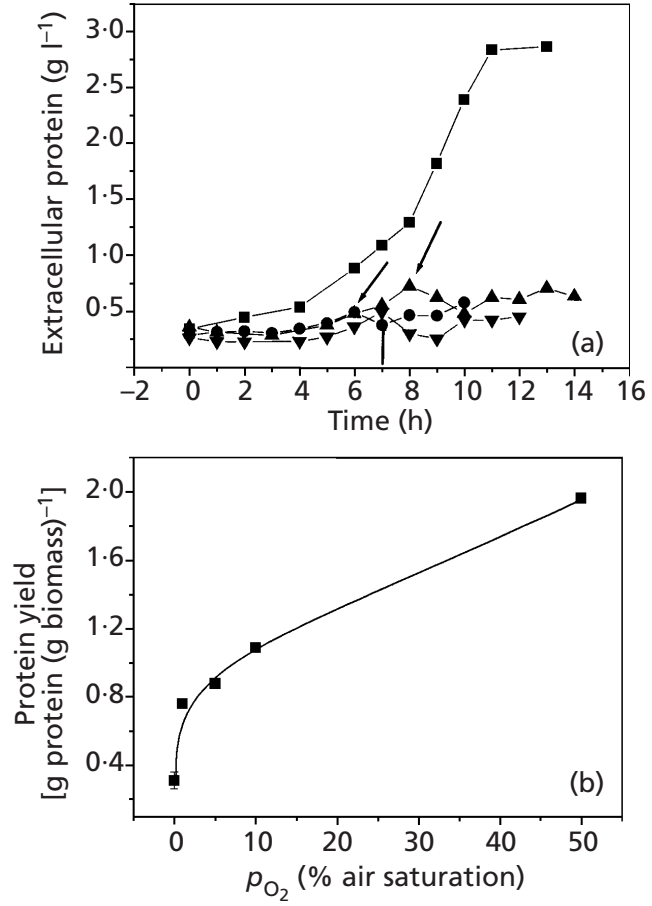

Fig. 6. (a) Effect of different $\mathrm{pO}_{2}$ levels on the production of extracellular proteins by $P$. aeruginosa PAO1. Arrows indicate the onset of oxygen limitation. $\mathbf{\square}, 1 \%$ of air saturation; , $5 \%$ of air saturation; $\boldsymbol{\Delta}, 10 \%$ of air saturation; $\boldsymbol{\nabla}, 50 \%$ of air saturation. (b) Protein yield for each of the different $\mathrm{pO}_{2}$ levels tested.

these conditions. As inferred from other mucoid strains of $P$. aeruginosa, the mucoid polysaccharide layer present on the surface of $P$. aeruginosa PAO1 is very likely to consist primarily of alginate (Mathee et al., 1999). The occurrence of this layer in controlled microaerobic and aerobic cultures of $P$. aeruginosa PAO1 seems to represent a physiological response of this strain to oxidative stress.

\section{Release of extracellular proteins by strain PAO1 when grown under different $\mathrm{pO}_{2}$ levels}

Fig. 6 shows the effects of different $p \mathrm{O}_{2}$ levels on the concentration of total excreted proteins and on the protein yield based on biomass formation during the period of controlled $p \mathrm{O}_{2}$. The concentration of extracellular proteins (Fig. 6a) paralleled the growth curve of PAO1 (Fig. 2a) in $p \mathrm{O}_{2}$-controlled batch culture to a

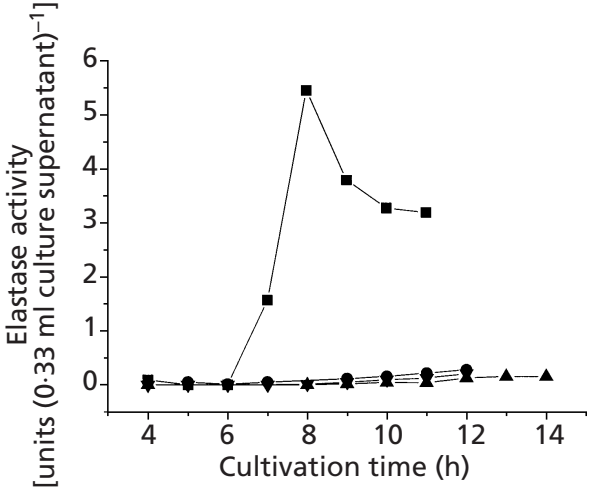

Fig. 7. Elastase activity as a function of the different $\mathrm{pO}_{2}$ levels tested during cultivation. $\square, 1 \%$ of air saturation;, $5 \%$ of air saturation; $\boldsymbol{\Delta}, 10 \%$ of air saturation; $\boldsymbol{\nabla}, 50 \%$ of air saturation.

certain extent, having the highest value at the lowest $p \mathrm{O}_{2}$ level studied ( $1 \%$ of air saturation). The secretion of proteins by PAO1 seemed to be considerably enhanced under microaerobic conditions. However, if the amount of secreted proteins based on biomass formed (referred to here as the protein yield) is considered, a different picture is obtained (Fig. 6b). The protein yield decreased from $1.97 \mathrm{~g}$ protein $\left(\mathrm{g}\right.$ biomass) ${ }^{-1}$ to $0.76 \mathrm{~g}$ protein $(\mathrm{g}$ biomass $)^{-1}$ as the $p \mathrm{O}_{2}$ level was reduced from 50 to $1 \%$ of air saturation. Interestingly, the protein yield from the oxygen-limited phase was nearly constant in the four independent PAO1 cultures. In all of these cultures, the protein yield dropped significantly after the onset of oxygen limitation and reached relatively low values of between 0.26 and $0.36 \mathrm{~g}$ protein $\left(\mathrm{g}\right.$ biomass) ${ }^{-1}$. Thus, the higher concentration of proteins secreted into the culture medium when an initial $p \mathrm{O}_{2}$ value of $1 \%$ of air saturation was used was due to the high biomass concentration. The specific secretion rate of proteins by PAO1 is, therefore, enhanced by the oxidative stress, as shown in Fig. 6(b).

$P$. aeruginosa is known to secrete a number of extracellular proteins as virulence factors; these include exoenzyme $S$, elastase, protease and phospholipase. In this work, we measured the activity of elastase in the PAO1 culture supernatants, to probe the homogeneity of the secreted proteins at different $p \mathrm{O}_{2}$ levels. Surprisingly, only microaerophilic growth conditions evoked the release of elastase by the cells (Fig. 7). No significant elastase activity was detected at higher dissolved oxygen 
concentrations (Fig. 7). Moreover, pyocyanine (the blue pigment produced by $P$. aeruginosa $\mathrm{PAO} 1$ ) production by PAO1 was also enhanced by the microaerophilic conditions (data not shown). Conversely, severe oxygen limitation seemed to reduce the release of elastase and other virulence factors by PAO1. In general, the release of some of these virulence factors by PAO1 is very sensitive to oxygen concentrations within the microaerobic range. More work is needed to study the pattern and dynamics of protein secretion by PAO1 under different oxidative stresses.

\section{DISCUSSION}

The results presented in this work show that $P$. aeruginosa exhibits several remarkable physiological responses to oxidative stress. First, in suspension culture, $P$. aeruginosa tends to strongly reduce the transfer rate of oxygen from the gas phase into the liquid phase, as revealed by measurements of the $k_{\mathrm{L}} a$ value (Fig. 2). Due to the strong decline in the $k_{\mathrm{L}} a$ value, oxygenlimited or microaerophilic conditions prevailed in the culture after a few hours incubation, even in a vigorously agitated bioreactor at a high aeration rate with pure oxygen. P. aeruginosa PAO1 clearly prefers a microaerophilic growth environment, producing its highest growth rate at the lowest controlled $p \mathrm{O}_{2}$ value $(1 \%$ of air saturation). A high concentration of oxygen in the growth medium appears to exert a stress upon $P$. aeruginosa PAO1, leading to a reduced growth rate, a longer lag phase and a greater release of proteins per gram of biomass formed.

Microaerobic conditions are known to dominate in biofilms, the preferred mode of growth for $P$. aeruginosa in the lungs of CF patients (Costerton et al., 1999; de Beer et al., 1994; Xu et al., 1998). Microaerobic conditions have also been reported to be optimal for the growth of $P$. aeruginosa on hydrocarbons (Chayabutra $\& \mathrm{Ju}, 2000)$. The preference of $P$. aeruginosa for microaerophilic growth is in accordance with recent findings from genomic analysis of this species (Croft $e t$ al., 2000). Analysis of the genomic DNA sequences of $P$. aeruginosa $\mathrm{PAO} 1$ has shown that this strain possesses a $15 \mathrm{~kb}$ cluster of genes that encodes a micro-oxic respiration system, similar to that used by nitrogenfixing bacteria, and which includes genes that encode homologues of nitrogen fixation/micro-oxic regulatory cascade proteins $(f i x L, f i x J$ and $f i x K / a n r)$. These genes enable nitrogen-fixing bacteria such as $A$. vinelandii to grow microaerobically and they may help $P$. aeruginosa to survive the unusual conditions in biofilms.

A second remarkable physiological and morphological change in $P$. aeruginosa PAO1 under oxidative stress is the occurrence of a polysaccharide on the cell surface (Fig. 3). This polysaccharide may be primarily composed of alginate, as found for several other strains of $P$. aeruginosa (Krieg et al., 1986; Mathee et al., 1999). In fact, alginate was also released into the culture broth by PAO1. Earlier studies have shown that $P$. aeruginosa PAO1 converts to the mucoid phenotype when cultures are treated with levels of $\mathrm{H}_{2} \mathrm{O}_{2}$ similar to those that can be normally released by polymorphonuclear leukocytes in the pulmonary tract of CF patients (Mathee et al., 1999). The strong oxygen limitation observed in liquid cultures of PAO1 may explain the non-mucoid appearance of this strain in cells from shake-flask cultures (Fig. 5a).

The known pathogenic roles of alginate in $P$. aeruginosa, in the form of a mucoid bacterial coating or as a free substance, have been reviewed by several authors (e.g. Govan \& Deretic, 1996; Miller \& Britigan, 1997). Besides its possible roles in adhesion and colonization (biofilm formation), this polysaccharide layer may also function as a physical barrier against the diffusion of oxygen into the cells, as has been suggested for the alginate-forming bacterium A. vinelandii (Sabra et al., 2000). A possible taxonomic relationship between the nitrogen-fixing bacterium $A$. vinelandii and the opportunistic pathogen $P$. aeruginosa has been discussed previously (Fyfe \& Govan, 1983). A common feature of $A$. vinelandii and $P$. aeruginosa is the formation of the microbial polysaccharide alginate by these organisms under certain conditions (Sabra et al., 2001, 1999).

Another important role of the exopolysaccharide of $P$. aeruginosa is the avoidance of encounters with phagocyte-derived reactive oxygen intermediates (Miller \& Britigan, 1997). The formation of reactive oxygen and reactive nitrogen intermediates is an important innate immune response of host cells upon their infection by microbial pathogens (Bogdan et al., 2000; Nathan \& Shiloh, 2000). Pathogenic bacteria have developed several mechanisms of defence against these toxic reactive intermediates. In addition to the formation of a polysaccharide coat, other non-enzymic and enzymic means of removing these intermediates from the surroundings have been discussed for different pathogens (Miller \& Britigan, 1997). For example, P. aeruginosa has been reported to have highly specific enzymic pathways for oxidant inactivation, including those catalysed by superoxide dismutase, catalase and peroxidase. In this work, we postulate a possible new and more efficient defence strategy of pathogen cells against oxidants and related nitric oxide derivatives. For the first time, we have shown that $P$. aeruginosa $\mathrm{PAO} 1$ can strongly reduce oxygen transfer to the culture, causing oxygen limitation and thus simply blocking the sources for the formation of reactive oxygen intermediates. It would be interesting to investigate whether $P$. aeruginosa $\mathrm{PAO} 1$ could also cause oxygen limitation in its surrounding environment in the lungs of CF patients by such a mechanism, thus avoiding the toxicity of reactive oxygen species. In fact, $P$. aeruginosa $\mathrm{PAO} 1$ itself grows best under these oxygen-limited (microaerobic) conditions and seems to be more toxic (see below).

A third remarkable physiological response of $P$. aeruginosa PAO1 to oxidative stress is its enhanced release of proteins (Fig. 6b). The mechanisms and intrinsic physiological consequences of these enhanced secretions are basically unknown. So far, little work has been done on this enhanced protein secretion, particularly with re- 
spect to the release of different virulence factors as a response to oxidative stress. Indeed, the identification of these excreted proteins will be interesting, since the proteins excreted under different $p \mathrm{O}_{2}$ levels are highly heterogeneous. For example, elastase activity was almost only recorded at the lowest $\mathrm{pO}_{2}$ value tested (Fig. 7). Moreover, the formation of pyocyanine by PAO1 was also enhanced by the microaerophilic conditions. This pigment can inhibit the capacity of macrophages to produce reactive nitrogen intermediates without altering cell viability (Shellito et al., 1992). Culture supernatants are currently being examined for their toxicity towards mammalian cells and analysed by two-dimensional electrophoresis for identification of the proteins secreted at different dissolved oxygen concentrations. Preliminary studies with supernatants taken from two different cultures $\left(p \mathrm{O}_{2}=1\right.$ and $\left.10 \%\right)$ of $P$. aeruginosa showed considerable differences in the toxicity of these supernatants towards mammalian cell cultures (Bi et al., 2001). This variable toxicity may be due to the formation of different proteins by the two cultures, as revealed by two-dimensional gel analysis (unpublished data).

In conclusion, $P$. aeruginosa $\mathrm{PAO} 1$ prefers microaerobic conditions for growth and can create these environmental conditions itself through at least two mechanisms: (i) blockage of oxygen transfer and (ii) formation of a polysaccharide layer. In particular, the blockage of oxygen transfer by this species may represent a new and efficient mechanism for the defence of the organism against reactive oxygen and nitrogen intermediates or it may be relevant to the host-pathogen relationship. More research is needed to investigate the mechanism(s) of blockage of oxygen transfer and the pathogenicity of $P$. aeruginosa under microaerobic conditions.

\section{ACKNOWLEDGEMENTS}

We gratefully acknowledge the help of Dr H. Lünsdorf for taking the electron microscopy pictures of cells. We would also like to thank Angela Walter for her excellent assistance in the sample analysis and Dr H. Biebl for critical reading of the manuscript.

\section{REFERENCES}

Bailey, J. E. \& Ollis, D. F. (1985). Biochemical Engineering Fundamentals, 2nd edn. New York: McGraw-Hill.

Bi, J.-X., Wirth, M., Beer, C., Sabra, W. \& Zeng, A.-P. (2001). The use of a C-FOS-GFP reporter system for monitoring apoptosis of animal cells induced by exotoxins of Pseudomonas aeruginosa. In Proceedings of the 17th Meeting of the European Society for Animal Cell Technology, June 10-14, Tylösand, Sweden.

Bogdan, C., Röllinghoff, M. \& Diefenbach, A. (2000). Reactive oxygen and reactive nitrogen intermediates in innate and specific immunity. Curr Opin Immunol 12, 64-76.

Chayabutra, C. \& Ju, L.-K. (2000). Degradation of n-hexadecane and its metabolites by Pseudomonas aeruginosa under microaerobic and anaerobic conditions. Appl Environ Microbiol 66, 493-498.

Chayabutra, C., Wu, J. \& Ju, L.-K. (2001). Rhamnolipid production by Pseudomonas aeruginosa under denitrification: effects of limiting nutrients and carbon substrate. Biotechnol Bioeng 72, 25-33.

Costerton, J. W., Stewart, P. S. \& Greenberg, E. P. (1999). Bacterial biofilms: a common cause of persistent infections. Science 284, 1318-1332.

Croft, L., Beatson, S. A., Whitchurch, C. B., Huang, B., Blakeley, R. L. \& Mattick, J. S. (2000). An interactive web-based Pseudomonas aeruginosa genome database: discovery of new genes, pathways and structures. Microbiology 146, 2351-2364.

de Beer, D., Stoodley, P. \& Lewandowski, Z. (1994). Effects of biofilm structures on oxygen distribution and mass transport. Biotechnol Bioeng 43, 1131-1138.

Deretic, V., Martin, D. W., Schurr, M. J., Mudd, M. H., Hibler, N. S., Curcic, R. \& Boucher, J. C. (1993). Conversion to mucoidy in Pseudomonas aeruginosa. Biotechnology 11, 1133-1136.

Fyfe, J. A. M. \& Govan, J. R. W. (1983). Synthesis, regulation and biological function of bacterial alginate. Prog Ind Microbiol 18, 45-83.

Geckil, H., Stark, B. C. \& Webster, D. A. (2001). Cell growth and growth uptake of Escherichia coli and Psendomonas aeruginosa are differently affected by the genetically engineered Vitreoscilla hemoglobin gene. J Biotechnol 85, 57-66.

Goldberg, J. B. \& Pier, G. B. (2000). The role of CFTR in susceptibility to Pseudomonas aeruginosa infections in cystic fibrosis. Trends Microbiol 8, 514-520.

Govan, J. R. W. \& Deretic, V. (1996). Microbial pathogenesis in cystic fibrosis: mucoid Pseudomonas aeruginosa and Burkbolderia cepacia. Microbiol Rev 60, 539-574.

Govan, J. R. W. \& Harris, G. S. (1986). Pseudomonas aeruginosa and cystic fibrosis: unusual bacterial adaptation and pathogenesis. Microbiol Sci 3, 302-308.

Greenberg, E. P. (2000). Bacterial genomics. Pump up the versatility. Nature 406, 947-948.

Guerra-Santos, A., Kaeppeli, L. H. \& Fiechter, O. (1986). Dependence of Pseudomonas aeruginosa continuous culture biosurfactant production on nutritional and environmental factors. Appl Microbiol Biotechnol 24, 443-448.

Haba, E., Espuny, M. J., Busquets, M. \& Manresa, A. (2000). Screening and production of rhamnolipids by Pseudomonas aeruginosa 47T2 NCIB 40044 from waste frying oils. J Appl Microbiol 88, 379-387.

Ishida, H., Ishida, Y., Kurosaka, Y., Otani, T., Sato, K. \& Kobayashi, H. (1998). In vitro and in vivo activities of levofloxacin against biofilm-producing Pseudomonas aeruginosa. Antimicrob Agents Chemother 42, 1641-1645.

Kamath, S., Kapatral, V. \& Chakrabarty, A. M. (1998). Cellular function of elastase in Pseudomonas aeruginosa: role in the cleavage of nucleoside diphosphate kinase and in alginate synthesis. Mol Microbiol 30, 933-941.

Kessler, E., Safrin, M., Olson, J. C. \& Ohman, D. E. (1993). Secreted LasA of Pseudomonas aeruginosa is a staphylolytic protease. J Biol Chem 268, 7503-7508.

Kharazmi, A. (1991). Mechanisms involved in the evasion of the host defence by Pseudomonas aeruginosa. Immunol Lett 30, 201-205.

Knutson, C. A. \& Jeanes, A. (1968). Determination of the composition of uronic acid mixtures. Anal Biochem 24, 482-490.

Kock, A. K., Käppeli, O., Fiechter, A. \& Reiser, J. (1991). Hydrocarbon assimilation and biosurfactant production in Pseudomonas aeruginosa mutants. J Bacteriol 173, 4212-4219.

Krieg, D. P., Bass, J. A. \& Mattingly, S. J. (1986). Aeration selects 
for mucoid phenotype of Pseudomonas aeruginosa. J Clin Microbiol 24, 986-990.

Leitäo, J. H. \& Sä-Correia, I. (1997). Oxygen-dependent upregulation of transcription of alginate genes $\operatorname{alg} A$, $\operatorname{alg} C$ and $\operatorname{alg} D$ in Pseudomonas aeruginosa. Res Microbiol 148, 37-43.

Maier, R. M. \& Soberon-Chavez, G. (2000). Pseudomonas aeruginosa rhamnolipids: biosynthesis and potential applications. Appl Microbiol Biotechnol 54, 625-633.

Mathee, K., Ciofu, O., Sternberg, C. \& 9 other authors (1999). Mucoid conversion of Pseudomonas aeruginosa by hydrogen peroxide: a mechanism for virulence activation in the cystic fibrosis lung. Microbiology 145, 1349-1357.

Mian, F. A., Jarman, T. R. \& Righelato, R. C. (1978). Biosynthesis of exopolysaccharide by Pseudomonas aeruginosa. J Bacteriol 134, 418-422.

Miller, R. A. \& Britigan, B. E. (1997). Role of oxidants in microbial pathophysiology. Clin Microbiol Rev 10, 1-18.

Nathan, C. \& Shiloh, M. U. (2000). Reactive oxygen and nitrogen intermediates in the relationship between mammalian hosts and microbial pathogens. Proc Natl Acad Sci U S A 97, 8841-8848.

Pearson, J. P., Pesci, E. C. \& Iglewski, B. H. (1997). Roles of Pseudomonas aeruginosa las and $r h l$ quorum-sensing systems in control of elastase and rhamnolipid biosynthesis genes. J Bacteriol 179, 5756-5767.

Pier, G. B. (1998). Pseudomonas aeruginosa: a key problem in cystic fibrosis. ASM News 64, 339-347.

Rocha, C., San-Blas, F., San-Blas, G. \& Vierma, L. (1992). Biosurfactant production by two isolates of Pseudomonas aeruginosa. World J Microbiol Biotechnol 8, 125-128.

Rumbaugh, K. P., Griswold, J. A., Iglewski, B. H. \& Hamood, A. N. (1999). Contribution of quorum sensing to the virulence of Pseudomonas aeruginosa in burn wound infections. Infect Immun 67, 5854-5862.
Sabra, W., Zeng, A.-P., Sabry, S., Omar, S. \& Deckwer, W.-D. (1999). Effect of phosphate and oxygen concentrations on alginate production and stoichiometry of metabolism of Azotobacter vinelandii under microaerobic conditions. Appl Microbiol Biotechnol 52, 773-780.

Sabra, W., Zeng, A.-P., Lünsdorf, H. \& Deckwer, W.-D. (2000). Effect of oxygen on formation and structure of Azotobacter vinelandii alginate and its role in protecting nitrogenase. Appl Environ Microbiol 66, 4037-4044.

Sabra, W., Zeng, A.-P. \& Deckwer, W.-D. (2001). Bacterial alginate: physiology, product quality and process aspects. Appl Microbiol Biotechnol 56, 315-325.

Sato, S., Mukataka, S., Kataoka, H. \& Takahashi, J. (1984). Effects of pressure and dissolved oxygen concentration on growth of Pseudomonas aeruginosa. J Ferment Technol 62, 71-75.

Shellito, J., Nelson, S. \& Sorensen, R. U. (1992). Effect of pyocyanine, a pigment of Pseudomonas aeruginosa, on production of reactive nitrogen intermediates by murine alveolar macrophages. Infect Immun 60, 3913-3915.

Stanbury, P. F. \& Whitaker, A. (1987). Principles of Fermentation Technology. Edited by P. F. Stanbury \& A. Whitaker. Oxford: Pergamon.

Xu, K. D., Stewart, P. S., Xia, F., Huang, C.-T. \& McFeters, G. A. (1998). Spatial physiological heterogeneity in Pseudomonas aeruginosa biofilm is determined by oxygen availability. Appl Environ Microbiol 64, 4035-4039.

Zaborina, O., Dhiman, N., Ling Chen, M., Kostal, J., Holder, I. A. \& Chakrabarty, A. M. (2000). Secreted products of a nonmucoid Pseudomonas aeruginosa strain induce two modes of macrophage killing: external-ATP-dependent, PZZ-receptor-mediated necrosis and ATP-independent, caspase-mediated apoptosis. Microbiology 146, 2521-2530.

Received 11 January 2002; revised 26 April 2002; accepted 11 June 2002. 